\title{
Histórico de Consumo de Álcool como Fator Preditivo de Sobrevida em Pacientes com Carcinoma de Células Escamosas de Boca e Orofaringe: Follow-up de 15 Anos
}

doi: https://doi.org/10.32635/2176-9745.RBC.2020v66n1.573

\author{
Alcohol Consumption History as a Predictive Factor of Survival in Patients with Mouth and Oropharyngeal Squamous Cell \\ Carcinoma: Follow-up of 15 Years \\ Historia del Consumo de Alcohol como Factor Predictivo para la Supervivencia en Pacientes con Carcinoma de Células \\ Escamosas Bucales y Orofaríngeas: Seguimiento de 15 Años
}

Paulo Goberlânio de Barros Silva'; Isabel Leite Soares²; Felipe Herbert de Oliveira Mendes ${ }^{3}$; Clarissa Sales de Paula Campêlo; Maria do Perpétuo Socorro Saldanha de Cunha5; Mário Rogério Lima Mota6; Thinali Sousa Dantas; Fabrício Bitu Sousa ${ }^{8}$

\begin{abstract}
Resumo
Introduçáo: $\mathrm{O}$ carcinoma de células escamosas é o tumor de maior importância na região de cabeça e pescoço, em razão da sua incidência e mortalidade. Sabe-se que fatores como consumo de álcool estáo relacionados à diminuiçáo da sobrevida de tumores tanto estimulando a progressão tumoral como causando comorbidades importantes, sendo um fator relevante para estudo. Objetivo: Avaliar a influência do histórico de álcool em características clinicoprognósticas de pacientes com carcinoma de células escamosas de boca e orofaringe (CCEBO). Método: Estudo de coorte, retrospectivo, no qual 156 prontuários de pacientes etilistas e 78 prontuários de pacientes não etilistas com CCEBO diagnosticados no Hospital Haroldo Juaçaba, em Fortaleza, Ceará, foram avaliados, entre 2000 e 2014, para análise de dados como idade, sexo, raça, localizaçáo do tumor, estadiamento TNM, tratamentos realizados e sobrevida em 15 anos por meio dos testes $\mathrm{X}^{2}$, Long-Rank e modelos de regressão multinomial e de Cox (SPSS 20,0; $<<0,05)$. Resultados: Houve maior prevalência de homens entre os pacientes etilistas ( $p<0,001)$, com tumores T3-T4 ( $p=0,003)$, linfonodos positivos $(\mathrm{p}=0,006)$ que realizaram tratamentos paliativos $(\mathrm{p}<0,001)$ e menor prevalência abaixo de 65 anos $(\mathrm{p}<0,001)$, quando havia histórico familiar de câncer $(\mathrm{p}=0,043)$. A sobrevida dos pacientes etilistas foi menor $(\mathrm{p}=0,040)$ e os fatores que diminuíram a sobrevida de maneira independente foram sexo masculino $(\mathrm{p}=0,042)$, estadiamento T3-T4 ( $\mathrm{p}=0,004)$, metástase linfonodal $(\mathrm{p}=0,012)$, idade $>65$ anos $(\mathrm{p}=0,035)$ e localização na língua $(p=0,042)$. O sexo masculino foi independentemente associado ao etilismo $(p<0,001)$. Conclusáo: $O$ álcool é um fator de prognóstico em pacientes com CCEBO, mostrando maior prevalência em pacientes T3-T4 e, assim, influenciando negativamente no prognóstico. Palavras-chave: Carcinoma de Células Escamosas; Sobrevida; Transtornos Relacionados ao Uso de Álcool.
\end{abstract}

\section{Abstract}

Introduction: Squamous cell carcinoma is the most important tumor in the head and neck region, due to its incidence and mortality. It is known that factors as alcohol consumption are related to the decrease of the survival of tumors, either stimulating tumor progression or causing considerable comorbidities, being an important study factor. Objective: Evaluate the influence of alcohol history on clinical and prognostic characteristics of patients with mouth oropharynx squamous cell carcinoma (MOSCC). Method: A retrospective cohort study in which 156 charts of alcoholic patients and 78 medical charts of non-alcoholic patients with MOSCC diagnosed at Haroldo Juaçaba Hospital in Fortaleza, state of Ceará were evaluated between 2000 and 2014 for data analysis such as age, gender and race, tumor location, TNM staging, treatments performed and 15 -year survival through $\mathrm{X}^{2}$, Long-Rank and Cox and multinomial regression models (SPSS 20.0; $\mathrm{p}<0.05)$. Results: Men were more prevalent among alcoholic patients ( $<0.001)$, with $\mathrm{T} 3 / 4$ tumors $(\mathrm{p}=0.003)$, positive lymph nodes $(\mathrm{p}=0.006)$ who submitted to palliative treatments $(\mathrm{p}<0.001)$ and lower prevalence under 65 years $(p<0.001)$, when there was a family history of cancer $(p=0.043)$. The survival of alcoholic patients was lower $(\mathrm{p}=0.040)$ and the factors that independently reduced survival were male sex $(\mathrm{p}=0.042), \mathrm{T} 3-\mathrm{T} 4$ staging $(\mathrm{p}$ $=0.004)$, lymph node metastasis $(\mathrm{p}=0.012)$, age $>65$ years $(\mathrm{p}=0.035)$ and tumor in the tongue $(\mathrm{p}=0.042)$. Male sex was independently associated with alcohol consumption $(p<0.001)$. Conclusion: Alcohol is a prognostic factor in patients with MOSCC, showing a higher prevalence in T3-T4 patients and, thus, negatively influencing the prognosis.

Key words: Carcinoma, Squamous Cell; Survival; Alcohol-Related Disorders.
Resumen

Introducción: El carcinoma de células escamosas es el tumor de mayor importancia en la región de cabeza y cuello, debido a su incidencia y mortalidad. Se sabe que factores como el consumo de alcohol están relacionado con la disminución de la supervivencia de tumores tanto estimulando la progresión tumoral, como provocando comorbilidades considerables, siendo un factor de estudio importante. Objetivo: Evaluar la influencia del historial del consumo de alcohol en las características clínicas-pronósticas de pacientes con carcinoma de células escamosas oral y orofaringe (CCEOO). Método: Estudio retrospectivo en el que se evaluaron 156 registros médicos de pacientes consumidores de alcohol y 78 de pacientes no alcohólicos con CCEOO diagnosticados en el Hospital Haroldo Juaçaba, en Fortaleza, Ceará, entre 2000 y 2014, para el análisis de datos como edad, sexo raza, escolaridad, los antecedentes familiares, vínculo matrimonial, registro en el servicio, ubicación del tumor, clasificación de TNM, los tratamientos realizados y la supervivencia durante 15 ańos a través de las pruebas X² Long-Rank y modelos de regresión multinomial y de Cox (SPSS 20.0; $\mathrm{p}<0,05)$. Resultados: Hubo una mayor prevalencia de hombres entre pacientes alcohólicos $(\mathrm{p}<0,001)$, con tumores T3-T4 $(\mathrm{p}=0,003)$, ganglios linfáticos positivos $(\mathrm{p}=0,006)$, y realizó tratamientos paliativos $(\mathrm{p}<0,001)$ y menor prevalencia en paciente menores de 65 ańos ( $\mathrm{p}<0,001)$; cuando se presentaron antecedentes familiares de cáncer $(\mathrm{p}=0,043)$. La supervivencia de los pacientes alcohólicos fue menor $(\mathrm{p}=0,040)$; $y$ los factores que disminuyeron la supervivencia de forma independiente fueron hombres $(\mathrm{p}=0,042)$; estadificación $\mathrm{t} 3-\mathrm{t} 4(\mathrm{p}=0,004)$; metástasis a ganglios linfáticos $(\mathrm{p}=0,012)$; edad $>65$ ańos $(\mathrm{p}=0,035)$; localización de la lengua $(\mathrm{p}=0,042)$. El sexo masculino se asoció independientemente con el consumo de alcohol $(\mathrm{p}<0,001)$. Conclusión: El alcohol define el pronóstico en pacientes con CCEOO, muestra una mayor prevalencia en pacientes con T3-T4, por lo tanto, influye negativamente en el pronóstico.

Palabras clave: Carcinoma de Células Escamosas; Sobrevida; Trastornos Relacionados con Alcohol.

${ }^{1}$ Centro Universitário Christus (Unichristus). Universidade Federal do Ceará (UFC). Fortaleza (CE), Brasil. Orcid iD: https://orcid.org/0000-0002-1513-9027

${ }^{2}$ Unichristus. Fortaleza (CE), Brasil. Orcid iD: https://orcid.org/0000-0002-7593-0080

${ }^{3}$ Unichristus. Fortaleza (CE), Brasil. Orcid iD: https://orcid.org/0000-0002-6048-2086

${ }^{4}$ Unichristus. Fortaleza (CE), Brasil. Orcid iD: https://orcid.org/0000-0002-5622-7314

${ }^{5}$ Escola Cearense de Oncologia. Hospital Haroldo Juaçaba. Fortaleza (CE), Brasil. Orcid iD: https://orcid.org/0000-0003-0682-6869

${ }^{6}$ UFC. Fortaleza (CE), Brasil. Orcid iD: https://orcid.org/0000-0003-3778-0584

${ }^{7}$ Unichristus. UFC. Fortaleza (CE), Brasil. Orcid iD: https://orcid.org/0000-0003-2543-3315

${ }^{8}$ Unichristus. UFC. Fortaleza (CE), Brasil. Orcid iD: https://orcid.org/0000-0002-6430-9475

Endereço para correspondência: Mário Rogério Lima Mota. Laboratório de Patologia Oral. Departamento de Clínicas Odontológicas da UFC. Rua Monsenhor Furtado, s/n - Porangabussu. Fortaleza (CE), Brasil. CEP 60430-350. E-mail: mariolmota@yahoo.com.br 


\section{INTRODUÇÃO}

Entre os tumores malignos, o carcinoma de células escamosas (CCE) destaca-se com principal tipo histológico em cavidade oral e orofaringe. As taxas de incidência e mortalidade variam em cada país, de acordo com as diferenças socioeconômicas, expectativas de vida, cultura, fatores ambientais, qualidade da assistência médica, hábitos, entre outros ${ }^{1}$.

O consumo de álcool foi primeiro determinado como um fator de risco para câncer de cavidade oral, orofaringe, hipofaringe e laringe pela Agência Internacional de Pesquisa sobre o Câncer (Iarc) ${ }^{2}$. E atualmente é reconhecido atuando por mecanismos que envolvem modulação de polimorfismos genéticos, do folato e na reparação do $\mathrm{DNA}^{2}$. As evidências ainda sugerem que a suscetibilidade genética também desempenha um papel importante aumentando a relaçáo do álcool com o desenvolvimento do câncer ${ }^{3,4}$.

Estima-se que, no Brasil, 11,2\% dos homens e $5,2 \%$ das mulheres são consumidores e dependentes de álcool ${ }^{5}$. Na cavidade oral, o etanol é oxidado em acetaldeído por bactérias da microbiota. Uma vez que o metabolismo adicional do acetaldeído-acetacetato é limitado por bactérias orais, a concentração de acetaldeído na saliva é 10 a 100 vezes maior do que no sangue ${ }^{6}$. Esse alto nível de acetaldeído tem contato direto com a mucosa, atuando como um solvente para vários outros carcinógenos introduzidos pelo tabagismo, dieta e produtos químicos nas células, especialmente na mucosa do trato aerodigestivo superior ${ }^{7}$.

O uso crônico de álcool representa um sério desafio para a saúde pública. No Reino Unido, em um estudo prospectivo realizado em 2018, há associação inversa entre consumo de álcool e sobrevida dos indivíduos com câncer de cabeça e pescoço ${ }^{8}$. Assim, visto que o álcool pode influenciar na sobrevida, o objetivo deste estudo é avaliar a influência do histórico de consumo de álcool na sobrevida de pacientes com câncer de boca e de orofaringe.

\section{MÉTODO}

Trata-se de um estudo de coorte, retrospectivo, no qual foram selecionados 234 prontuários de pacientes com CCE em cavidade oral e orofaringe assistidos no Hospital Haroldo Juaçaba do Instituto do Câncer do Ceará, de 2000 a 2014. Todos os prontuários deveriam conter informações acerca do uso prévio de álcool, sendo selecionados, assim, 156 prontuários de pacientes com histórico de uso de álcool e 78 sem histórico (relação expostos: não expostos de aproximadamente $2: 1$ ).

Foram analisados dados sociodemográficos como idade, sexo, raça, escolaridade, histórico familiar, vínculo matrimonial do paciente, histórico de tabagismo, tipo de entrada no hospital (Sistema Único de Saúde - SUS ou privado e planos de saúde), bem como dados clínicos, tipo histológico do tumor, sítio anatômico, TNM, estadiamento do tumor e tratamento realizado (cirurgia; cirurgia associada à radioterapia; radioterapia; radioterapia associada à quimioterapia; cirurgia associada à radioterapia e à quimioterapia; cirurgia associada à quimioterapia ou a nenhum tratamento).

A sobrevida foi obtida por meio da diferença entre a data do início do tratamento (dia, mês e ano) e a data do óbito (dia, mês e ano) utilizando para fins de análise estatística a quantidade de semanas entre essas datas?.

Os dados foram analisados no software Statistical Package for the Social Sciences (SPSS) versão 20.0 para Windows, adotando uma confiança de $95 \%$ e utilizando os testes exato de Fisher, qui-quadrado de Pearson, Long-Rank Mantel Cox para análises bivariadas e modelos de regressáo logística multinomial, e regressão de Cox para análise multivariada.

Foram respeitados todos os preceitos éticos de aceitação no Comitê de Ética em Pesquisa do Centro Universitário Christus e do Hospital Haroldo Juaçaba sob o número 2.191.839, emitido em 28 de julho de 2017.

\section{RESULTADOS}

Foram selecionados para este estudo um total de 156 pacientes não etilistas e 78 etilistas, um estudo de coorte, retrospectivo, de status não expostos: expostos de 2:1 (Tabela 1).

$\mathrm{Na}$ amostra, dos 156 pacientes estudados com CCE de boca e orofaringe, 108 (46,2\%) foram do sexo feminino e a maioria era do sexo masculino $(53.8 \%)$, com pacientes que possuíam histórico de álcool significativamente mais associados ao sexo masculino $(\mathrm{p}=<0,001)$. A idade de maior ocorrência foi de até 65 anos de idade $(\mathrm{n}=123$, $52,6 \%$ casos), sem diferença significante entre os grupos de pacientes etilistas e não etilistas $(\mathrm{p}=<0,001)$. A maioria dos pacientes era de raça parda $(\mathrm{n}=163,69,2 \%)$ e com o primeiro grau completo $(\mathrm{n}=149,63,7 \%)$ (Tabela 1$)$.

O sítio de maior acometimento do CCE de boca e orofaringe foi a língua com $\mathrm{n}=118(50,4 \%)$ casos, tanto para pacientes etilistas $(\mathrm{n}=34,43,6 \%)$ como para pacientes não etilistas $(n=53,8 \%)(p=84)$. Com relação ao TNM, os pacientes etilistas apresentaram maior número de casos T3-T4 ( $\mathrm{n}=54,69,2 \%)$ do que os pacientes não etilistas $(\mathrm{n}=24,30,8 \%) \quad(\mathrm{p}=0,003)$. A presença de metástases linfonodais também foi mais prevalente nos pacientes etilistas $(\mathrm{n}=56,71,8 \%)$ do que os pacientes não etilistas $(\mathrm{n}=55,47,8 \%)(\mathrm{p}=0,006)$. Porém, não houve diferença significativa entre a presença de metástases a distância entre os dois grupos de estudo (Tabela 1). 
Tabela 1. Distribuição dos pacientes etilistas e não etilistas em diferentes variáveis abordadas. Tempo médio de sobrevida de pacientes etilistas e não etilistas

\begin{tabular}{|c|c|c|c|c|c|c|c|}
\hline \multirow{2}{*}{ Variáveis } & \multirow{2}{*}{\multicolumn{2}{|c|}{ Total }} & \multicolumn{4}{|c|}{ Etilismo } & \multirow{2}{*}{ p-Valo } \\
\hline & & & \multicolumn{2}{|c|}{ Não etilista } & \multicolumn{2}{|c|}{ Etilista } & \\
\hline \multicolumn{8}{|l|}{ Sexo } \\
\hline Feminino & 108 & $46,2 \%$ & $100 *$ & $64,1 \%$ & 8 & $10,3 \%$ & $<0,001$ \\
\hline Masculino & 126 & $53,8 \%$ & 56 & $35,9 \%$ & $70^{*}$ & $89,7 \%$ & \\
\hline \multicolumn{8}{|l|}{ Idade } \\
\hline Até 65 anos & 123 & $52,6 \%$ & 69 & $44,2 \%$ & $54^{*}$ & $69,2 \%$ & $<0,001$ \\
\hline Mais de 65 anos & 111 & $47,4 \%$ & $87^{*}$ & $55,8 \%$ & 24 & $30,8 \%$ & \\
\hline \multicolumn{8}{|l|}{ Raça } \\
\hline Parda & 163 & $69,7 \%$ & 109 & $69,9 \%$ & 54 & $69,2 \%$ & 0,920 \\
\hline Não parda & 71 & $30,3 \%$ & 47 & $30,1 \%$ & 24 & $30,8 \%$ & \\
\hline \multicolumn{8}{|l|}{ Escolaridade } \\
\hline Analfabeto & 58 & $24,8 \%$ & 44 & $28,2 \%$ & 14 & $17,9 \%$ & 0,230 \\
\hline $1^{\circ} \mathrm{grau}$ & 149 & $63,7 \%$ & 95 & $60,9 \%$ & 54 & $69,2 \%$ & \\
\hline $2^{\circ}$ grau ou médio & 27 & $11,5 \%$ & 17 & $10,9 \%$ & 10 & $12,8 \%$ & \\
\hline \multicolumn{8}{|l|}{ Localização } \\
\hline Língua & 118 & $50,4 \%$ & 84 & $53,8 \%$ & 34 & $43,6 \%$ & 0,246 \\
\hline Assoalho bucal & 35 & $15,0 \%$ & 22 & $14,1 \%$ & 13 & $16,7 \%$ & \\
\hline Paredes orofaringe & 38 & $16,2 \%$ & 20 & $12,8 \%$ & 18 & $23,1 \%$ & \\
\hline Palato & 33 & $14,1 \%$ & 24 & $15,4 \%$ & 9 & $11,5 \%$ & \\
\hline Outros & 10 & $4,3 \%$ & 6 & $3,8 \%$ & 4 & $5,1 \%$ & \\
\hline \multicolumn{8}{|l|}{$\mathrm{T}$} \\
\hline $\mathrm{T} 1-\mathrm{T} 2$ & 86 & $43,9 \%$ & $62^{*}$ & $52,5 \%$ & 24 & $30,8 \%$ & 0,003 \\
\hline T3-T4 & 110 & $56,1 \%$ & 56 & $47,5 \%$ & $54^{*}$ & $69,2 \%$ & \\
\hline \multicolumn{8}{|l|}{$\mathrm{N}$} \\
\hline NO & 77 & $39,9 \%$ & $55^{*}$ & $47,8 \%$ & 22 & $28,2 \%$ & 0,006 \\
\hline $\mathrm{N}+$ & 116 & $60,1 \%$ & 60 & $52,2 \%$ & $56^{*}$ & $71,8 \%$ & \\
\hline \multicolumn{8}{|l|}{ Tratamento } \\
\hline Nenhum & 34 & $14,5 \%$ & $31^{*}$ & $19,9 \%$ & 3 & $3,8 \%$ & $<0,001$ \\
\hline Cirurgia & 16 & $6,8 \%$ & $16^{*}$ & $10,3 \%$ & 0 & $0,0 \%$ & \\
\hline Cirurgia + radioterapia & 34 & $14,5 \%$ & $28^{*}$ & $17,9 \%$ & 6 & $7,7 \%$ & \\
\hline Radioterapia & 52 & $22,2 \%$ & 35 & $22,4 \%$ & 17 & $21,8 \%$ & \\
\hline Cirurgia + radioterapia + quimioterapia & 23 & $9,8 \%$ & 11 & $7,1 \%$ & $12^{*}$ & $15,4 \%$ & \\
\hline Radioterapia + quimioterapia & 75 & $32,1 \%$ & 35 & $22,4 \%$ & $40^{*}$ & $51,3 \%$ & \\
\hline \multicolumn{8}{|l|}{ Vínculo matrimonial } \\
\hline Não & 195 & $83,3 \%$ & 134 & $85,9 \%$ & 61 & $78,2 \%$ & 0,137 \\
\hline Sim & 39 & $16,7 \%$ & 22 & $14,1 \%$ & 17 & $21,8 \%$ & \\
\hline \multicolumn{8}{|l|}{ Histórico familiar } \\
\hline Não & 164 & $70,1 \%$ & 116 & $74,4 \%$ & $48^{*}$ & $61,5 \%$ & 0,043 \\
\hline Sim & 70 & $29,9 \%$ & $40^{*}$ & $25,6 \%$ & 30 & $38,5 \%$ & \\
\hline \multicolumn{8}{|l|}{ Tabagismo } \\
\hline Não & 166 & $70,9 \%$ & $133^{*}$ & $85,3 \%$ & 33 & $42,3 \%$ & $<0,001$ \\
\hline Sim & 68 & $29,1 \%$ & 23 & $14,7 \%$ & $45^{*}$ & $57,7 \%$ & \\
\hline \multicolumn{8}{|l|}{ Entrada } \\
\hline SUS & 112 & $77,8 \%$ & 71 & $74,0 \%$ & 41 & $85,4 \%$ & 0,119 \\
\hline Não SUS & 32 & $22,2 \%$ & 25 & $26,0 \%$ & 7 & $14,6 \%$ & \\
\hline
\end{tabular}


Houve uma alta taxa de tratamentos considerados paliativos. A maior parte dos casos foi tratada com radioterapia e quimioterapia $(\mathrm{n}=75,32,1 \%)$ seguido de radioterapia $(n=52,22,2 \%)$. O tipo de tratamento realizado nos pacientes etilistas foi significantemente cirurgia, radioterapia e quimioterapia $(\mathrm{n}=12,15,4 \%)$ e radioterapia e quimioterapia $(n=40,51,3 \%)$, enquanto os pacientes sem histórico de consumo de álcool apresentaram maior frequência de tratamentos cirúrgicos $(\mathrm{n}=16,10,3 \%)$, cirurgia + radioterapia $(\mathrm{n}=28,17,9 \%)$, ou ausência de tratamento $(\mathrm{n}=31,19,9 \%)(\mathrm{p}=<0,001 \%)$ (Tabela 1).

Ausência de vínculo matrimonial ocorreu mais vezes nos pacientes tanto etilistas $(\mathrm{n}=61,78,2 \%)$ como não etilistas $(\mathrm{n}=134,85,9 \%)(\mathrm{p}=0,137)$, assim como a entrada no serviço pelo SUS $(\mathrm{n}=41,85,4 \%$ e $\mathrm{n}=71,74,0 \%$, respectivamente) ( $\mathrm{p}=0,119 \%)$; porém, os pacientes com histórico familiar de câncer apresentaram associação inversa com o histórico de consumo de álcool ( $\mathrm{p}=0,043)$, mostrando maior prevalência nos pacientes não etilistas $(\mathrm{n}=40,25,6 \%)$ que nos etilistas $(\mathrm{n}=30,38,4 \%)$. O histórico de fumo, por sua vez, foi diretamente associado ao consumo de álcool ( $\mathrm{p}=0,001)$ (Tabela 1$)$.

O tempo médio de sobrevida dos pacientes com histórico de consumo de álcool $(58,89+7,73)$ foi significantemente inferior aos pacientes sem histórico de consumo de álcool $(88,23 \pm 6,14)(\mathrm{p}=0,40)$ (Figura 1), apresentando, ao final do período avaliado, uma taxa de sobrevida de 44,9\% (Tabela 2).

A taxa de sobrevida dos pacientes do sexo masculino $(n=58)$ foi menor nos pacientes do sexo feminino $(n=65)$. Idade, raça, escolaridade e localização da lesão não influenciaram na sobrevida dos pacientes em 15 anos.

Quando estudados os dados clinicopatológicos, observa-se que pacientes que apresentaram tumores com tamanhos T1-T2 tiveram aproximadamente 89,78 meses de sobrevida $(\mathrm{n}=56)$, diferentemente dos pacientes em T3T4 com 71,39 meses de vida $(\mathrm{n}=50)$. Os pacientes que não apresentavam metástase para linfonodo apresentaram 48 meses de sobrevida, já, nos que apresentaram metástase linfonodal, a sobrevida caiu para $49,1 \%$. O tratamento, vínculo matrimonial, histórico familiar, tabagismo e entrada ou não pelo SUS não apresentaram significância (Tabela 2).

Os fatores associados ao histórico de consumo de álcool foram, respectivamente, tabagismo e sexo (Tabela 3).

O tabagismo esteve associado ao histórico de álcool $(15,66)$ e o sexo masculino obteve associação de 11,38

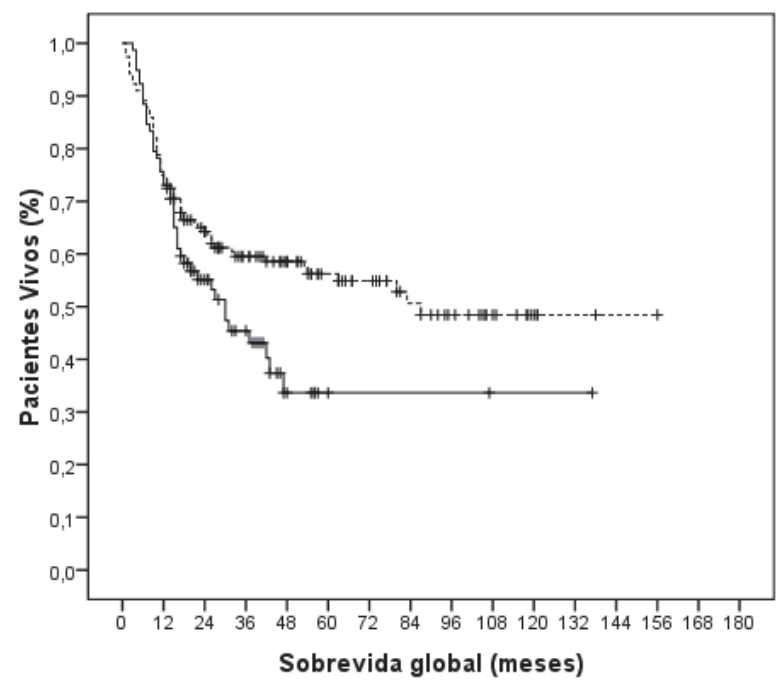

Figura 1. Curva de sobrevida global em 15 anos de acompanhamento do grupo de pacientes com câncer de boca e orofaringe atendidos e tratados no Hospital Haroldo Juaçaba, categorizados mediante histórico prévio de etilismo

Legendas: ${ }^{*} \mathrm{p}=0,002$, teste de Long-Rank Mantel-Cox. Linha tracejada $=$ pacientes năo etilistas; Linha năo tracejada $=$ pacientes etilistas.

Tabela 2. Sobrevida em 15 anos de pacientes etilistas e não etilistas, atendidos no Hospital Haroldo Juaçaba com CCE de boca e orofaringe, entre 2000 e 2004

\begin{tabular}{|c|c|c|c|c|}
\hline \multirow{2}{*}{ Variáveis } & \multicolumn{3}{|c|}{ Sobrevida em 15 anos } & \multirow{2}{*}{ p-valor } \\
\hline & Tempo (meses) & $\mathbf{N}$ & $\%$ & \\
\hline Sobrevida global & $80,86 \pm 5,15$ & 123 & $52,6 \%$ & - \\
\hline \multicolumn{5}{|l|}{ Uso de álcool } \\
\hline Não etilista & $88,23 \pm 6,14$ & 88 & $56,4 \%$ & 0,040 \\
\hline Etilista & $58,89 \pm 7,73$ & $35^{*}$ & $44,9 \%$ & \\
\hline \multicolumn{5}{|l|}{ Sexo } \\
\hline Feminino & $93,37 \pm 7,44$ & 65 & $60,2 \%$ & 0,042 \\
\hline Masculino & $63,42 \pm 5,89$ & $58 *$ & $46,0 \%$ & \\
\hline \multicolumn{5}{|l|}{ Idade } \\
\hline Até 65 anos & $74,20 \pm 6,06$ & 67 & $54,5 \%$ & 0,348 \\
\hline Mais de 65 anos & $79,42 \pm 7,28$ & 56 & $50,5 \%$ & \\
\hline
\end{tabular}


Tabela 2. continuação

\begin{tabular}{|c|c|c|c|c|}
\hline \multirow{2}{*}{ Variáveis } & \multicolumn{3}{|c|}{ Sobrevida em 15 anos } & \multirow{2}{*}{ p-valor } \\
\hline & Tempo (meses) & $\mathbf{N}$ & $\%$ & \\
\hline \multicolumn{5}{|l|}{ Raça } \\
\hline Parda & $65,24 \pm 4,50$ & 85 & $52,1 \%$ & 0,921 \\
\hline Não parda & $82,29 \pm 9,23$ & 38 & $53,5 \%$ & \\
\hline \multicolumn{5}{|l|}{ Escolaridade } \\
\hline Analfabeto & $49,70 \pm 6,25$ & 23 & $39,7 \%$ & 0,061 \\
\hline $1^{\circ}$ grau & $83,35 \pm 6,51$ & 81 & $54,4 \%$ & \\
\hline $2^{\circ}$ grau ou médio & $94,54 \pm 12,53$ & 19 & $70,4 \%$ & \\
\hline \multicolumn{5}{|l|}{ Localização } \\
\hline Língua & $84,77 \pm 6,25$ & 73 & $61,9 \%$ & 0,065 \\
\hline Assoalho bucal & $77,73 \pm 12,41$ & 17 & $48,6 \%$ & \\
\hline Paredes da orofaringe & $53,86 \pm 9,54$ & 17 & $44,7 \%$ & \\
\hline Palato & $43,19 \pm 7,06$ & 12 & $36,4 \%$ & \\
\hline Outros & $49,80 \pm 15,10$ & 4 & $40,0 \%$ & \\
\hline \multicolumn{5}{|l|}{$\mathrm{T}$} \\
\hline $\mathrm{T} 1-\mathrm{T} 2$ & $89,78 \pm 7,09$ & 56 & $65,1 \%$ & 0,004 \\
\hline T3-T4 & $71,39 \pm 7,21$ & $50^{*}$ & $45,5 \%$ & \\
\hline \multicolumn{5}{|l|}{$\mathrm{N}$} \\
\hline No & $98,00 \pm 8,37$ & 48 & $62,3 \%$ & 0,012 \\
\hline$N+$ & $60,59 \pm 5,48$ & $57^{*}$ & $49,1 \%$ & \\
\hline \multicolumn{5}{|l|}{ Tratamento } \\
\hline Nenhum & $50,03 \pm 9,26$ & 14 & $41,2 \%$ & 0,053 \\
\hline Cirurgia & $89,78 \pm 11,82$ & 12 & $75,0 \%$ & \\
\hline Cirurgia + radioterapia & $100,07 \pm 12,54$ & 22 & $64,7 \%$ & \\
\hline Radioterapia & $56,46 \pm 7,95$ & 24 & $46,2 \%$ & \\
\hline Cirurgia + radioterapia + quimioterapia & $61,63 \pm 10,36$ & 13 & $56,5 \%$ & \\
\hline Radioterapia + quimioterapia & $71,98 \pm 7,55$ & 38 & $50,7 \%$ & \\
\hline \multicolumn{5}{|l|}{ Vínculo matrimonial } \\
\hline Não & $82,58 \pm 5,65$ & 104 & $53,3 \%$ & 0,565 \\
\hline Sim & $65,60 \pm 10,75$ & 19 & $48,7 \%$ & \\
\hline \multicolumn{5}{|l|}{ Histórico familiar } \\
\hline Não & $81,71 \pm 6,05$ & 86 & $52,4 \%$ & 0,932 \\
\hline Sim & $69,59 \pm 8,51$ & 37 & $52,9 \%$ & \\
\hline \multicolumn{5}{|l|}{ Tabagismo } \\
\hline Não & $84,46 \pm 5,94$ & 89 & $53,6 \%$ & 0,278 \\
\hline Sim & $63,81 \pm 8,55$ & 34 & $50,0 \%$ & \\
\hline \multicolumn{5}{|l|}{ Entrada } \\
\hline SUS & $67,97 \pm 5,40$ & 61 & $54,5 \%$ & 0,922 \\
\hline Não SUS & $69,83 \pm 11,29$ & 17 & $53,1 \%$ & \\
\hline
\end{tabular}

Legendas: ${ }^{*} \mathrm{p}<0,05$, teste de Long-Rank Mantel Cox (média $\left.\pm E P M\right)$. 
Tabela 3. Análise multivariada de fatores associados ao histórico de consumo de álcool em pacientes com câncer de boca e orofaringe diagnosticados e tratados no Hospital Haroldo Juaçaba

\begin{tabular}{l|c|c|c|c}
\multicolumn{1}{c|}{ Variáveis } & p-Valor & HR ajustado & \multicolumn{2}{c}{ IC 95\% } \\
\hline Etilismo & & & \multicolumn{2}{c}{} \\
\hline Sexo (Masculino) & $<\mathbf{0 , 0 0 1}$ & $\mathbf{1 1 , 3 8}$ & $\mathbf{2 , 9 8}$ & $\mathbf{4 3 , 4 4}$ \\
\hline Idade (>65 anos) & 0,802 & - & - & - \\
\hline Raça (Pardo) & 0,583 & - & - & - \\
\hline Escolaridade (Analfabeto) & 0,105 & - & - & - \\
\hline Localização (Assoalho) & 0,489 & - & - & - \\
\hline T (T3-T4) & 0,137 & - & - & - \\
\hline N (N+) & 0,480 & - & - & - \\
\hline Tratamento (Cirúrgico) & 0,084 & - & - & - \\
\hline Vínculo matrimonial (Sim) & 0,690 & - & - & - \\
\hline Histórico familiar (Sim) & 0,851 & - & - & - \\
\hline Tabagismo (Sim) & $<0,001$ & $\mathbf{1 5 , 6 6}$ & $\mathbf{3 , 6 1}$ & $\mathbf{6 7 , 9 7}$ \\
\hline Entrada (SUS) & 0,132 & - & - & - \\
\hline
\end{tabular}

Legendas: * $<<0,05$, regressăo logística multinomial. $\mathrm{HR}=$ Hazard risk; IC = Intervalo de confiança.

ao etilismo. Os demais fatores, idade ( $>65$ anos), raça, escolaridade, localização, T3-T4, acometimento linfonodal, tratamento cirúrgico, vínculo matrimonial, histórico familiar, tabagismo e entrada (SUS) não apresentaram relevância estatística.

Entretanto, os fatores que influenciam a sobrevida dos pacientes foi a idade acima de 65 anos (odds ratio=2,14), a localização em língua (odds ratio=1,26), estágios T3-T4 (odds ratio $=2,78$ ), sendo o estadiamento $T$ o fator que mais fortemente aumenta o risco de morte. Coincidentemente, os pacientes T3-T4 tiveram histórico de álcool. As demais variáveis como sexo, etilismo, raça (não pardo), escolaridade (analfabeto), acometimento linfonodal, tratamento, vínculo matrimonial, histórico familiar, tabagismo, entrada (não SUS) não apresentaram fator estatisticamente significante (Tabela 4).

Tabela 4. Sobrevida em 15 anos de pacientes etilistas e não etilistas, atendidos no Hospital Haroldo Juaçaba, com CCE de boca e orofaringe entre 2000 e 2004

\begin{tabular}{|c|c|c|c|c|}
\hline Variáveis & p-Valor & HR & \multicolumn{2}{|c|}{ IC $95 \%$} \\
\hline Etilismo (Sim) & 0,298 & - & - & - \\
\hline Sexo $(M)$ & 0,159 & - & - & - \\
\hline Idade (>65) & 0,035 & 2,14 & 1,06 & 4,35 \\
\hline Raça (Não pardo) & 0,136 & - & - & - \\
\hline Escolaridade (Analfabeto) & 0,981 & - & - & - \\
\hline Localização (Língua) & 0,042 & 1,26 & 1,01 & 1,58 \\
\hline $\mathrm{T}(3 / 4)$ & 0,011 & 2,78 & 1,27 & 6,09 \\
\hline$N(N+)$ & 0,682 & - & - & - \\
\hline Tratamento (Cirurgia) & 0,699 & - & - & - \\
\hline Vínculo matrimonial (Sim) & 0,731 & - & - & - \\
\hline Histórico familiar (Sim) & 0,855 & - & - & - \\
\hline Tabagismo (Sim) & 0,454 & - & - & - \\
\hline Entrada (Não SUS) & 0,154 & & & \\
\hline
\end{tabular}

Legendas: ${ }^{*} \mathrm{p}<0,05$, modelo de regressão de Cox. HR = Hazar risk; IC = Intervalo de confiança. 


\section{DISCUSSÃO}

Aproximadamente metade da populaçáo adulta mundial consome bebidas alcoólicas, sendo o consumo de álcool um dos fatores de risco mais importantes para a saúde pública e a terceira principal causa de morte em alguns países ${ }^{10}$. Além disso, vários outros fatores, como antecedentes genéticos, gênero, idade, etnia, dieta, hormônios, bem como tabagismo e obesidade, podem ter impacto na modulação da resposta do organismo ao álcoo ${ }^{11}$. Estima-se que, mundialmente, quase metade da população com 15 anos ou mais $(44,5 \%)$ nunca consumiu álcool e cerca de $43 \%$ da população são bebedores atuais (consumiram nos últimos 12 meses).

É importante considerar isso para a região da cabeça e pescoço, visto que há associação ao aumento do risco de câncer cavidade oral e faringe, potencializando o efeito de iniciadores para a carcinogênese como o tabaco ${ }^{12}$. Observou-se que o histórico de consumo de álcool esteve associado ao pior prognóstico em pacientes com câncer de boca e orofaringe. Paciente etilistas apresentavam maior prevalência do sexo masculino ${ }^{13}$, estimativas similares também foram demonstradas pela Organização Mundial da Saúde em $2018^{14}$.

A maioria dos estudos, a partir do ano de 2014, relatou que indivíduos entre 60 e 64 anos e 65 anos ou mais exibiam um maior consumo atual de bebidas alcoólicas, relacionando-se também ao indivíduo do sexo masculino ${ }^{15}$.

A relação entre o consumo de bebidas alcoólicas e variáveis educacionais é raramente investigada, principalmente porque a identificação da relação causa-efeito requer realização de estudos prospectivos ${ }^{16}$, corroborando a falta de associaçáo entre consumo de álcool e escolaridade, que não acontece em uma série de estudos.

Pacientes etilistas também apresentaram maiores tumores e metástases linfonodais, tendo, portanto, como maioria, na modalidade de tratamento utilizada, a radioterapia combinada com quimioterapia. Além de agir como um fator de risco para a carcinogênese, estudos epidemiológicos também demonstraram que o consumo de álcool estava associado à invasão e metástase do câncer, levando a um mau prognóstico ${ }^{17}$; sabe-se que o estágio tumoral é um dos fatores prognósticos mais bem conhecidos.

Neste estudo, o histórico de álcool foi inversamente associado com histórico familiar de câncer, reforçando a etiopatogenia extrínseca do CEC de boca e orofaringe ${ }^{13}$, mas foi independentemente associado com uso de cigarros, que é um dos principais fatores sinérgicos para o desenvolvimento desse tipo de tumor ao sexo masculino, principal consumidor dessa associaçãa ${ }^{17}$.
O consumo de álcool é um fator de risco estabelecido e também um possível fator prognóstico para o carcinoma espinocelular de cabeça e pescoço, sendo esses resultados ainda controversos ${ }^{18,19}$. Neste estudo, a sobrevida global dos pacientes com histórico de consumo de álcool foi significantemente inferior, controversamente descrito por Schimansky et al. ${ }^{17}$ e semelhante ao demostrado por Beynon et al. ${ }^{8}$.

Outros estudos observaram também que dois terços das mortes por câncer relacionado ao álcool (boca, faringe, laringe, esôfago) acontecem em homens e um terço, em mulheres ${ }^{8,20}$.

A sobrevida de pacientes do sexo masculino foi significativamente menor, assim como aqueles que tiveram metástase linfonodal, no entanto, os fatores independentemente associados à menor sobrevida global foram os estádios T3-T4, idade acima de 65 anos e localização em língua. Esses achados ratificam o estudo de Jiang et al. ${ }^{21}$, que trouxe como resultados associaçóes mais fortes entre o consumo de álcool e a mortalidade por câncer encontradas particularmente entre aqueles com idade entre 50 e 69 anos.

Além disso, tem sido descrito que o acometimento da língua é considerado um sítio prognóstico ao CEC oral em razão da propensão para metástase linfonodal regional por meio da rede linfática ${ }^{22}$, sendo esta fortemente associada à recidiva pós-tratamento e à baixa sobrevida.

Apesar de o consumo de álcool náo ter se demonstrado uma variável independentemente associada ao pior prognóstico, o consumo excessivo e contínuo de álcool após o diagnóstico de câncer pode levar à diminuição da taxa de remissão da doença e ao aumento na incidência de cânceres secundários no fígado e trato aerodigestivo superior com impacto indireto nas taxas de sobrevida ${ }^{23}$.

O álcool pode ser usado como um mecanismo de enfrentamento para aliviar a ansiedade e o medo em torno de um diagnóstico de câncer e, dada sua natureza aditiva, pode resultar em consumo prolongado, aumentado ou retomado após um curto período de abstinência ${ }^{24}$. Desde os estudos limitados até hoje, o gênero, a escolaridade, o tempo desde o tratamento, a gravidade da doença e o consumo de álcool mais pesado foram identificados como preditores de mudança no consumo de álcool em pacientes com câncer.

\section{CONCLUSÃO}

Este trabalho contribuiu para o melhor entendimento acerca da influência de hábitos deletérios, como o histórico de álcool, com a progressão do câncer de boca e orofaringe. Entretanto, o presente estudo apresenta limitaçóes, como a natureza de ser retrospectivo, baseado em prontuários, 
influenciado por viés de memória e preenchimento e não poder distinguir/analisar se houve ou não cessação do consumo de álcool após o diagnóstico/início do tratamento.

O histórico de consumo de álcool em pacientes com CEC de boca e orofaringe está diretamente associado ao histórico de fumo, ao sexo masculino e a tumores diagnosticados em estádios maiores, decrescendo a sobrevida de forma dependente da idade e da localização do tumor primário. Estudos que avaliem a influência da cessaçáo do consumo de álcool em pacientes com CEC de boca e orofaringe são necessários, a fim de avaliar o impacto do hábito durante e após o tratamento.

\section{CONTRIBUIÇÕES}

Paulo Goberlânio de Barros Silva, Maria do Perpétuo Socorro Saldanha de Cunha e Thinali Sousa Dantas contribuiram na concepçáo e desenho do trabalho; e aquisição, análise, interpretação dos dados da pesquisa. Isabel Leite Soares, Felipe Herbert de Oliveira Mendes, Clarissa Sales de Paula Campêlo, Mário Rogério Lima Mota e Fabrício Bitu Sousa participaram da redação e revisão crítica com contribuição intelectual. Todos os autores aprovaram a versão final para publicação.

\section{DECLARAÇÃO DE CONFLITO DE INTERESSES}

Nada a declarar.

\section{FONTES DE FINANCIAMENTO}

Não há.

\section{REFERÊNCIAS}

1. Lydiatt WM, Patel SG, O'Sullivan B, et al. Head and neck cancers-major changes in the American Joint Committee on cancer eighth edition cancer staging manual. CA Cancer J Clin. 2017;67(2):122-37. doi: https://doi. org/10.3322/caac.21389

2. Islami F, Tramacere I, Rota M, et al. Alcohol drinking and laryngeal cancer: overall and dose-risk relation-a systematic review and meta-analysis. Oral Oncol. 2010;46(11):802-10. doi: https://doi.org/10.1016/j. oraloncology.2010.07.015

3. Oze I, Matsuo K, Wakai K, et al. Alcohol drinking and esophageal cancer risk: an evaluation based on a systematic review of epidemiologic evidence among the Japanese population. J Clin Oncol. 2011;41(5):677-92. doi: https://doi.org/10.1093/jjco/hyr026

4. Bagnardi V, Rota M, Botteri E, et al. Alcohol consumption and site-specific cancer risk: a comprehensive dose- response meta-analysis. Br J Cancer. 2015;112(3):58093. doi: https://doi.org/10.1038/bjc.2014.579

5. Petti S. Lifestyle risk factors for oral cancer. Oral Oncol.2009;45(4-5):340-50. doi: https://doi. org/10.1016/j.oraloncology.2008.05.018

6. Homann N, Jousimies-Somer H, Jokelainen K, et al. High acetaldehyde levels in saliva after ethanol consumption: methodological aspects and pathogenetic implications. Carcinogenesis. 1997;18(9):1739-43. doi: https://doi.org/10.1093/carcin/18.9.1739

7. Seitz HK, Stickel F. Molecular mechanisms of alcoholmediated carcinogenesis. Nat Rev Cancer. 2007;7(8):599612. doi: https://doi.org/10.1038/nrc2191

8. Beynon RA, Lang S, Schimansky S, et al. Tobacco smoking and alcohol drinking at diagnosis of head and neck cancer and all-cause mortality: results from head and neck 5000, a prospective observational cohort of people with head and neck cancer. Int J Cancer. 2018;143(5):1114-27. doi: https://doi.org/10.1002/ ijc.31416

9. Dantas TS, Silva PGB, Sousa EF, et al. Influence of educational level, stage, and histological type on survival of oral cancer in a Brazilian population: a retrospective study of 10 years observation. Medicine. 2016;95(3):e2314. doi: https://doi.org/10.1097/ MD.0000000000002314

10. Rehm J, Borges G, Gmel G, et al. The comparative risk assessment for alcohol as part of the Global Burden of Disease 2010 study: what changed from the last study?. Int J Alcohol Drug Res. 2013;2(1):1-5. doi: https://doi. org/10.7895/ijadr.v2i1.132

11. Zhou Y, Zheng J, Li S, et al. Alcoholic beverage consumption and chronic diseases. Int J Environ Res Public Health. 2016;13(6):522. doi: https://doi. org/10.3390/ijerph13060522

12. Fortin A, Wang CS, Vigneault E. Influence of smoking and alcohol drinking behaviors on treatment outcomes of patients with squamous cell carcinomas of the head and neck. Int J Radiat Oncol Biol Phys. 2009;74(4):106269. doi: https://doi.org/10.1016/j.ijrobp.2008.09.021

13. Ness-Jensen E, Lagergren J. Tobacco smoking, alcohol consumption and gastro-oesophageal reflux disease. Best Pract Res Clin Gastroenterol. 2017;31(5):501-8. doi: https://doi.org/10.1016/j.bpg.2017.09.004

14. World Health Organization. Global status report on alcohol and health 2018 [Internet]. Geneva: World Health Organization; 2018. [cited 2019 Dec 15]. Available from: https://apps.who.int/iris/bitstream/han dle/10665/274603/9789241565639-eng.pdf?ua=1

15. Leung J, Chiu V, Connor JP, et al. Alcohol consumption and consequences in adolescents in 68 low and middleincome countries: a multi-country comparison of risks by sex. Drug Alcohol Depend. 2019;205:107520. doi: https://doi.org/10.1016/j.drugalcdep.2019.06.022 
16. Zenic N, Terzic A, Ostojic L, et al. Educational and sport factors as predictors of harmful alcohol drinking in adolescence: a prospective study in Bosnia and Herzegovina. Int J Public Health. 2019;64(2):185-194. doi: https://doi.org/10.1007/s00038-018-1168-5

17. Schimansky S, Lang S, Beynon R, et al. Association between comorbidity and survival in head and neck cancer: results from head and neck 5000. Head Neck. 2019;41(4):1053-62. doi: https://doi.org/10.1002/ hed. 25543

18. Sawabe M, Ito H, Oze I, et al. Heterogeneous impact of alcohol consumption according to treatment method on survival in head and neck cancer: a prospective study. Cancer Sci. 2017;108(1):91-100. doi: https://doi. org/10.1111/cas.13115

19. Duffy SA, Ronis DL, McLean S, et al. Pretreatment health behaviors predict survival among patients with head and neck squamous cell carcinoma. J Clin Oncol. 2009;27(12):1969-75. doi: https://doi.org/10.1200/ JCO.2008.18.2188

20. Parkin DM, Boyd L, Walker LC. The fraction of cancer attributable to lifestyle and environmental factors in the UK in 2010. Br J Cancer. 2011;105(2):S77-S81. doi: https://doi.org/10.1038/bjc.2011.489

21. Jiang H, Livingston MJ, Room R, et al. Temporal associations of alcohol and tobacco consumption with cancer mortality. JAMA Netw Open. 2018;1(3):e180713. doi: https://doi.org/10.1001/ jamanetworkopen.2018.0713

22. Amin G, Siegel M, Naimi T. National Cancer Societies and their public statements on alcohol consumption and cancer risk. Addiction. 2018;113(10):1802-08. doi: https://doi.org/10.1111/add.14254

23. Alton D, Eng L, Lu L, et al. Perceptions of continued smoking and smoking cessation among patients with cancer. J Oncol Pract. 2018;14(5):e269-e279. doi: https://doi.org/10.1200/JOP.17.00029

24. Allison PJ. Factors associated with smoking and alcohol consumption following treatment for head and neck cancer. Oral Oncol. 2001;37(6):513-20. doi: https:// doi.org/10.1016/S1368-8375(01)00015-X 